\title{
MS20-05 | Small-angle Scattering Study for Developing Alkaline Durable Imidazolium-Based Grafted Anion Exchange Membranes for Pt-Free Fuel Cells
}

Zhao, Yue (National Institutes for Quantum and Radiological Science and Technology, Takasaki, JPN)

Anion-exchange membranes (AEMs) have been regarded as an alternative to proton-exchange membranes (PEMs) in energy conversion devices, due to the advantage of saving expensive platinum catalysts. However, neither the molecular design nor the property understanding is sufficient for developing AEMs capable of practical fuel cell applications. It is crucial to thoroughly study the current AEMs in terms of their chemical/microscopic structures and membrane properties. We recently developed new grafted AEMs by radiation-induced grafting method, where imidazolium and styrene monomers were grafted into poly(ethylene-co-tetrafluoroethylene) (ETFE) base films. We have discovered the correlations between suitable membrane properties and morphologies using smallangle scattering technique. Our results show that: 1) semi-crystalline feature conserved from the base film offers good mechanical properties; 2) a clear micro-phase separation between conducting phase composed of graft polymers and water, and non-conducting phase promotes the conductivity; 3 ) a homogeneous conducting phase is more alkaline stable than a heterogeneous conducting phase, and the ion transport in the homogeneous phase is more efficient. This study contributes to a better understanding of the interplay between the structure and properties of the anion-exchange membranes that should make it possible to rationally design membranes with optimal properties. 